\title{
Tratamiento biológico de la artritis reumatoide en España. Análisis de impacto presupuestario de la utilización de certolizumab pegol
}

\author{
Álvaro Hidalgo • Renata Villoro - Alexandra Ivanova • \\ Alberto Morell · Pablo Talavera • Belén Ferro
}

Published online: 29 June 2013

(C) The Author(s) 2013. This article is published with open access at Springerlink.com

Resumen Objetivo Estimar el impacto presupuestario (IP) en España del uso combinado de certolizumab pegol (siguiendo un régimen fijo de dosis subcutáneas cada 2 semanas, tras una dosis de inducción en las semanas 0, 2 y 4) más metotrexato (MTX), como terapia para la artritis reumatoide (AR) de moderada a grave.

Métodos Se diseñó un modelo desde la perspectiva del Sistema Nacional de Salud (SNS) con un horizonte temporal de 5 años (2013-2017). Se estimó la población candidata a esta terapia frente a otras terapias biológicas para la AR ([abatacept, adalimumab, etanercept, infliximab, tocilizumab, golimumab y rituximab] $+[\mathrm{MTX}]$ ) a partir de información epidemiológica y la revisión de publicaciones científicas. Se incluyeron los costes: farmacológicos, de administración y de monitorización (año base 2013). Se realizó un análisis de sensibilidad univariante sobre el conjunto de variables con mayor incertidumbre y se aplicó una tasa de descuento anual del $3 \%$.

\footnotetext{
Á. Hidalgo $(\bowtie)$

Facultad de Ciencias Jurídicas y Sociales, Universidad de Castilla la Mancha, Cobertizo San Pedro Mártir, S/N, 45071 Toledo, España

e-mail: alvaro.hidalgo@uclm.es

R. Villoro · A. Ivanova

Instituto Max Weber, Madrid, España

A. Morell

Servicio Farmacia, Hospital La Princesa, Madrid, España

P. Talavera

Departamento Médico, UCB Pharma, Madrid, España

B. Ferro

Departamento de Farmacoeconomía, UCB Pharma, Madrid,

España
}

Resultados Con las asunciones hechas en el modelo, la cifra de pacientes tratados con certolizumab pegol evolucionaría de 1.943 pacientes en 2013 a 6.234 en 2017. El modelo estima que durante el período 2013-2017 la utilización de certolizumab pegol en España generaría unos ahorros globales medios por valor de 10,26 millones de euros. Dichos ahorros serían debidos fundamentalmente a la reducción de los costes de administración y la ausencia de necesidad de intensificación de dosis o ajustes por peso del paciente.

Conclusiones El tratamiento de los pacientes con AR de moderada a grave en España con certolizumab pegol permitiría ahorros en el SNS español en el período 2013-2017.

Palabras clave Certolizumab pegol $\cdot$ Impacto presupuestario · Evaluación económica · Artritis reumatoide

Abstract Objective To carry out the Budget Impact Analysis (BIA) associated with the introduction of certolizumab pegol (fixed subcutaneous dosing regimen of $200 \mathrm{mg}$ every 2 weeks, with a $400 \mathrm{mg}$ induction dose at weeks 0,2 , and 4) plus methotrexate (MTX), into the Spanish market of biological disease-modifying anti-rheumatic drugs (bDMARDs) for the management of moderate-to-severe rheumatoid arthritis (RA).

Methods A model was developed to assess the BIA from the Spanish National Health System (NHS) perspective for a 5-year period (2013-2017). Epidemiological data and scientific literature were used to estimate the RA population eligible for certolizumab pegol therapy versus other therapies ([abatacept, adalimumab, etanercept, infliximab, tocilizumab, golimumab y rituximab] + [MTX]) Total cost (administration, monitoring, ex-factory prices) and market share (MS) were considered and based on year 2013 data. In 
the base case, we assume a 3\% annual discount rate. A sensitivity analysis was performed for the set of variables associated with a significant uncertainty.

Results According to the model, the number of patients treated with certolizumab pegol will rise from 1,943 (2013) to 6,234 (2017). The use of certolizumab pegol results in annual budgetary net savings of $€ 10.26$ millions for the period 2013-2017. The savings are basically due to a reduction in administration costs and the absence of the need to intensify or adjust doses based on the patient's weight.

Conclusions Treating moderate-to-severe RA patients with certolizumab pegol would generate net savings for the Spanish NHS in the period 2013-2017.

Keywords Certolizumab pegol - Budget impact .

Economic evaluation $\cdot$ Rheumatoid arthritis

\section{Introducción}

La artritis reumatoide (AR) es una enfermedad inflamatoria crónica que cursa por brotes y que, a pesar de ser tratada, puede resultar en la destrucción, deformidad y discapacidad progresivas de las articulaciones, en una disminución de la calidad de vida y en un aumento de la mortalidad, sobre todo, por enfermedad cardiovascular en comparación con la población general [1]. En España, la AR afecta aproximadamente al $0,5 \%$ de la población adulta [2]. El tratamiento actual estándar para pacientes con AR y enfermedad persistente a pesar de un tratamiento agresivo tras el uso y fracaso de al menos un fármaco antirreumático modificador de la enfermedad (FAME) sintético convencional, consiste en la administración de FAME biológicos (bFAME), ya sea en monoterapia, ya en combinación con metotrexato (MTX), según se establece en el Consenso sobre el uso de terapias biológicas en la AR de la Sociedad Española de Reumatología (SER) [3, 4]. Sin embargo, se ha demostrado que una proporción importante de pacientes con AR de moderada a grave no responde de manera adecuada a los tratamientos actuales y continúa padeciendo episodios de actividad de la enfermedad mientras se encuentra bajo tratamiento [5].

Certolizumab pegol es un fármaco recombinante humano, antagonista del factor de necrosis tumoral alfa (TNF- $\alpha$ ), consistente en un fragmento Fab' de un anticuerpo monoclonal dirigido contra el TNF- $\alpha$ conjugado con dos moléculas de polietilenglicol (PEG) de $20 \mathrm{KDa}$ cada una, que se administra según un régimen fijo y estable de dosis subcutáneas de $200 \mathrm{mg}$ cada 2 semanas, con una dosis de inducción de $400 \mathrm{mg}$ en las semanas 0,2 y 4, en monoterapia o en combinación con MTX. Certolizumab pegol está indicado para el tratamiento de pacientes adultos con AR activa, de moderada a grave, cuya respuesta a FAME convencionales, incluyendo MTX, ha sido inadecuada $[5,6]$.
La eficacia y seguridad de certolizumab pegol en pacientes con AR de moderada a grave que no han respondido adecuadamente a un régimen de monoterapia con MTX han sido demostradas en los estudios clínicos RAPID 1, RAPID 2 y FAST4WARD, cuyos resultados indican que el tratamiento con certolizumab pegol ha conseguido una rápida y significativa reducción de los síntomas y signos de la AR [7-9].

Uno de los factores importantes para determinar la inclusión de certolizumab pegol en el mercado de FAME biológicos en España es el impacto que pueda tener la misma sobre el presupuesto del Sistema Nacional de Salud (SNS). Un análisis de impacto presupuestario (IP) consiste en la estimación cuantitativa del cambio previsto en el gasto sanitario debido a la introducción de un nuevo fármaco para la atención de una patología o grupo de pacientes concretos. Para cuantificar el impacto económico de la adopción de certolizumab pegol en el mercado español es necesario estimar primero el número de pacientes con AR susceptibles de utilizarlo, así como suponer una tasa de sustitución gradual de otros FAME biológicos actualmente prescritos. El objetivo principal del presente análisis de IP es proporcionar al decisor sanitario una estimación de la alteración en su factura sanitaria debido a la introducción de certolizumab pegol en el mercado de FAME biológicos.

\section{Material y métodos}

\section{Diseño}

Para estimar el IP asociado a la introducción de certolizumab pegol en el mercado español de FAME biológicos, hemos diseñado un modelo utilizando el programa de Microsoft Excel 2010, basándonos en las recomendaciones internacionales para este tipo de evaluaciones $[10,11]$. Las variables incluidas en el modelo han sido validadas por un panel de expertos, compuesto por un reumatólogo, un farmacéutico de hospital y expertos en Economía de la Salud.

La población española con AR de moderada a grave susceptible de ser tratada con terapias biológicas se estimó a partir de información epidemiológica y de la revisión de publicaciones científicas [12-15].

Los FAME biológicos incluidos en el modelo han sido los aprobados para el tratamiento de la AR moderada a grave en España y avalados por la Sociedad Española de Reumatología (SER) como tratamientos de primera o segunda línea tras la respuesta inadecuada a un FAME convencional [16]: anti-TNF- $\alpha$ + MTX [certolizumab pegol, adalimumab, etanercept, infliximab (3 y $5 \mathrm{mg} / \mathrm{kg}$ ), y golimumab], anti-linfocitos + MTX [abatacept y rituximab] y anti-interleuquina $6+$ MTX [tocilizumab]. La Tabla 1 
Tabla 1 Régimen de posología de las terapias

\begin{tabular}{|c|c|c|c|c|c|}
\hline & \multirow[t]{2}{*}{ Intervención } & \multirow[t]{2}{*}{ Dosis/Régimen } & \multirow{2}{*}{$\begin{array}{l}\text { mg por } \\
\text { administración }\end{array}$} & \multicolumn{2}{|c|}{ Número de administraciones } \\
\hline & & & & $\begin{array}{l}\text { Primeros } \\
\text { seis meses }\end{array}$ & $\begin{array}{l}\text { Siguientes } \\
\text { seis meses }\end{array}$ \\
\hline \multirow[t]{5}{*}{ Anti-TNF $\alpha$} & $\begin{array}{l}\text { Certolizumab pegol } \\
+ \text { MTX }\end{array}$ & $\begin{array}{l}400 \mathrm{mg} \text { en las semanas } 0,2 \text { y } \\
4, \text { y a continuación } 200 \mathrm{mg} \\
\text { cada } 2 \text { semanas }\end{array}$ & 200 & 16 & 13 \\
\hline & Etanercept + MTX & $25 \mathrm{mg} 2$ veces a la semana* & 25 & 52 & 52 \\
\hline & Adalimumab + MTX & $40 \mathrm{mg}$ en semanas alternas & 40 & 13 & 13 \\
\hline & Infliximab + MTX & $\begin{array}{l}3 \mathrm{mg} / \mathrm{kg} \text { o } 5 \mathrm{mg} / \mathrm{kg} \text { en las } \\
\text { semanas } 0,2,6 \text { y luego cada } \\
8 \text { semanas }\end{array}$ & $\begin{array}{l}\text { En función de } \\
\text { la distribución } \\
\text { de la masa } \\
\text { corporal }\end{array}$ & 5,5 & 3,25 \\
\hline & Golimumab + MTX & $50 \mathrm{mg}$ cada mes & 50 & 6 & 6 \\
\hline \multirow[t]{2}{*}{ Anti-linfocitos } & Abatacept + MTX & $\begin{array}{l}<60 \mathrm{~kg}: 500 \mathrm{mg}, 60-100 \mathrm{~kg} \text { : } \\
750 \mathrm{mg},>100 \mathrm{~kg}: 1 \mathrm{~g} \\
\text { repetido } 2 \text { y } 4 \text { semanas } \\
\text { después de la infusión } \\
\text { inicial, luego cada } 4 \\
\text { semanas. No se requiere } \\
\text { ajuste de dosis cuando se } \\
\text { utiliza en combinación con } \\
\text { otros FAME }\end{array}$ & $\begin{array}{l}\text { En función de } \\
\text { la distribución } \\
\text { de la masa } \\
\text { corporal }\end{array}$ & 7,5 & 6,5 \\
\hline & Rituximab + MTX & $\begin{array}{l}1 \mathrm{~g} \text {, repetido } 2 \text { semanas } \\
\text { (ciclo de } 2 \text { dosis); repetir el } \\
\text { ciclo cada } 6 \text { meses }\end{array}$ & 1.000 & 2,0 & 2,0 \\
\hline Anti-IL6 & Tocilizumab + MTX & $8 \mathrm{mg} / \mathrm{kg}$ cada 4 semanas & $\begin{array}{l}\text { En función de } \\
\text { la distribución } \\
\text { de la masa } \\
\text { corporal }\end{array}$ & 6,5 & 6,5 \\
\hline
\end{tabular}

Fuente: EMA [5]

* En la práctica clínica el régimen utilizado para etanercept puede consistir también en $50 \mathrm{mg}$ una vez a la semana. No se ha incluido en el modelo dicha variante dado que no afecta los resultados del impacto presupuestario. Anti-TNF-alfa: fármacos antagonistas del factor de necrosis tumoral alfa; Anti-IL6: fármaco antagonista de la interleuquina-6; MTX: metotrexato; mg: miligramos; kg: kilogramo; FAME: fármaco antirreumático modificador de la enfermedad

muestra el régimen de posología de las terapias incluidas en el modelo y la frecuencia de administración de los fár$\operatorname{macos}[5]$.

El diseño del modelo tiene en consideración que algunas de las terapias (certolizumab pegol, infliximab y abatacept) tienen un régimen de posología inicial seguido por un régimen de posología de mantenimiento. Para todos los fármacos, cualquier dosis administrada en la semana 27 fue considerada parte del segundo ciclo de seis meses, en lugar de ser parte del régimen de los primeros seis meses.

El modelo de IP realizado asume que la proporción de pacientes en primer año de tratamiento (con dosis de inicio en los primeros seis meses) es constante e igual al $10 \%$ cada año, que los pacientes permanecen en el mismo régimen de tratamiento y dosificación los cinco años de tratamiento y que las dosis de los fármacos podrán ajustarse según la masa corporal predeterminada.

\section{Perspectiva y horizonte temporal}

El IP asociado a la posible adopción de certolizumab pegol se analiza desde la perspectiva del SNS español y de la Consejería de Sanidad de las diversas Comunidades Autónomas (CCAA). Por lo tanto, se han incluido solamente los costes directos sanitarios, financiados públicamente, y se han excluido los costes directos no sanitarios, financiados por el paciente, así como los costes indirectos debidos a posibles pérdidas de productividad laboral. El horizonte temporal considerado es de cinco años, de 2013 a 2017; el año base para las variables principales del análisis ha sido el 2013 y la moneda: el euro (€).

Estimación de la población de pacientes susceptible de ser tratada. Caso base

Asumimos que la población susceptible de recibir certolizumab pegol es equivalente a la población con AR moderada- 
Figura 1 Pacientes elegibles para tratamientos con FAME biológicos, 2013-2017. Fuente: Elaboración propia a partir de datos de la SER [14], ENS [13] e INE [12, 15] AR: artritis reumatoide; FAME biológico (bFAME): fármaco antirreumático modificador de la enfermedad biológico

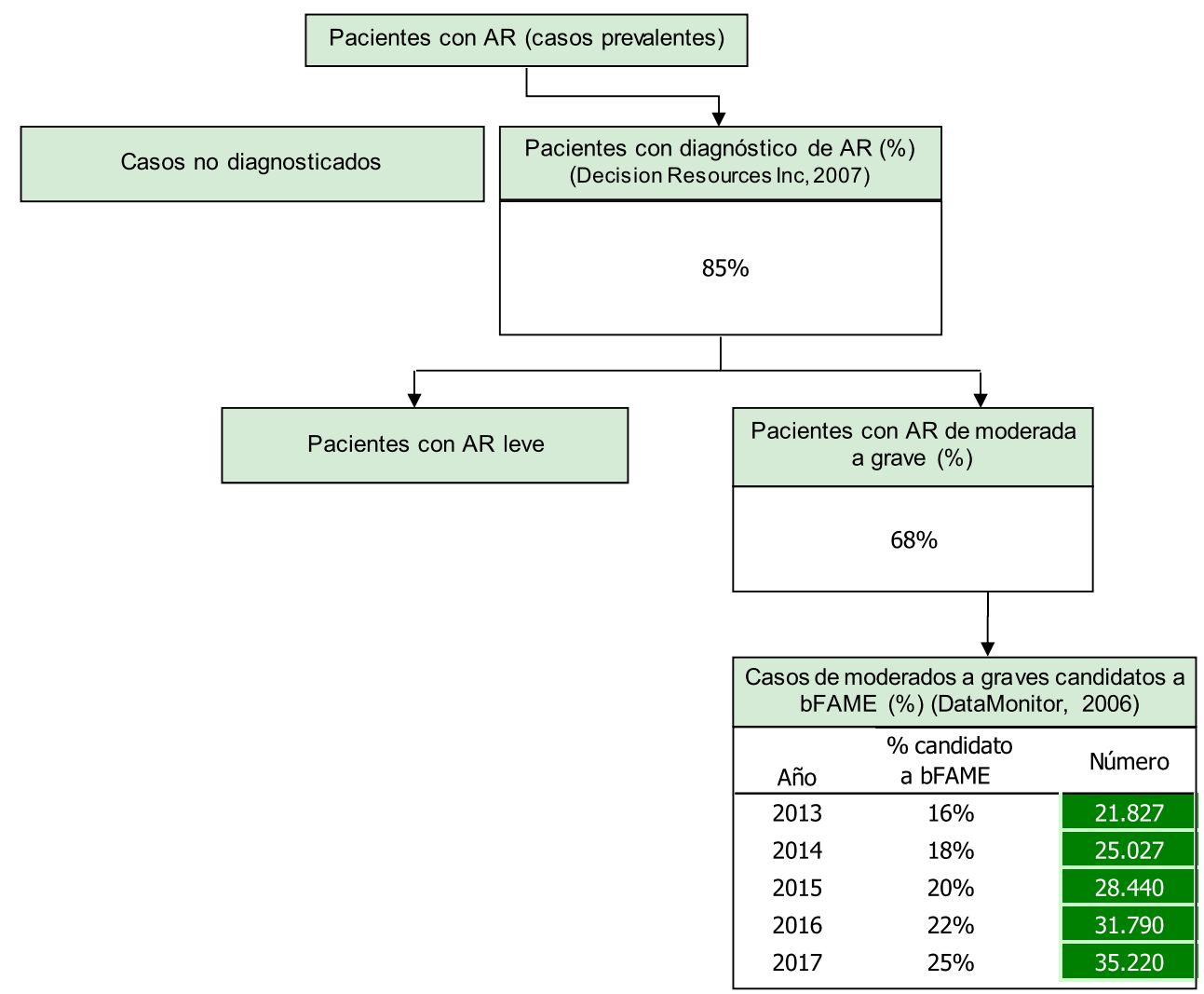

grave susceptible de ser tratada con algún FAME biológico para dicha indicación. Para su estimación, calculamos primero la prevalencia de la AR en la población española de 15 años o más a partir de datos de las Estimaciones de la Población Actual de España del Instituto Nacional de Estadística (INE) [12], de la última Encuesta Nacional de Salud (ENS) disponible [13] y de la SER [14]. Las proyecciones de población para los años 2013-2017 se tomaron de las proyecciones de población a corto plazo publicadas por el INE [15]. Asumimos que solo aquellos pacientes diagnosticados de AR son candidatos a ser tratados. Del porcentaje de casos diagnosticados, los porcentajes de casos de AR que son moderados o graves y la proporción de pacientes susceptibles de tratamiento con FAME biológicos de 2013 a 2015 se obtuvieron de análisis de mercado publicados [16].

Se asume que la proporción de pacientes diagnosticados y la proporción de pacientes con AR moderada o grave son constantes a lo largo del tiempo. La proporción de pacientes elegibles para tratamiento con FAME biológicos en 2016 y 2017 se obtiene de la extrapolación de las proporciones en los años 2013-2015. La Fig. 1 muestra el diagrama de decisión utilizado en el modelo para proyectar el número de pacientes elegibles en el período 2013-2017. En dicho período se espera que la cifra de pacientes elegibles para terapias biológicas pase de más de 21.000 a más de 35.000 pacientes a nivel nacional.
Estimación de los costes directos sanitarios. Caso base

Para estimar los costes directos sanitarios asociados a cada tratamiento, se han considerado los costes farmacológicos, los costes de administración y formación y los costes de monitorización (visitas médicas y análisis clínicos).

\section{Costes farmacológicos}

Los costes unitarios de los fármacos se obtuvieron a partir de bases de datos oficiales españolas para el año 2013 [17, 18], que corresponden a los precios establecidos por la Comisión Interministerial de Precios de Medicamentos. El precio de venta laboratorio (PVL) de la jeringa precargada de $200 \mathrm{mg}$ de certolizumab pegol es 474,00 EUR. Los PVL de adalimumab (40 mg), etanercept (25 mg) y golimumab (50 mg) son de 514,15 EUR, 118,40 EUR y 1.117,00 EUR, respectivamente, por jeringa precargada. Los precios por vial de abatacept $(250 \mathrm{mg})$, infliximab (100 $\mathrm{mg})$, rituximab (500 $\mathrm{mg}$ ) y tocilizumab $(80 \mathrm{mg})$ son de 334,82 EUR, 536,28 EUR, 1.247,00 EUR y 139,60 EUR, respectivamente. Para el caso base, el modelo aplica sobre estos precios, un $7,5 \%$ de descuento obligatorio según Real Decreto-Ley 8/2010 [19]. Suponemos que el coste de MTX es insignificante $(0,1$ EUR por comprimido) $\mathrm{y}$, por tanto, asumimos para el modelo un coste cero (Tabla 2). 
Tabla 2 Resumen de los costes medios por paciente/año según terapia

\begin{tabular}{|c|c|c|c|c|c|c|}
\hline \multirow[t]{2}{*}{ Medicamentos } & \multirow[t]{2}{*}{$\begin{array}{l}\text { Presentación/ } \\
\text { Dosis }\end{array}$} & \multicolumn{2}{|c|}{$\begin{array}{l}\text { Datos } \\
\text { administración }\end{array}$} & \multicolumn{3}{|c|}{$\begin{array}{l}\text { PVL }-7,5 \% \\
\text { (descuento obligatorio RD 8/2010) }\end{array}$} \\
\hline & & $\mathrm{mg}$ & Uds. & Por unidad & $\begin{array}{l}\text { Por } \\
\text { administración }\end{array}$ & Coste anual $^{\mathrm{a}}$ \\
\hline Certolizumab pegol + MTX & 2 jeringas/ $400 \mathrm{mg}$ & 200 & 1 & $438,45 €$ & $438,45 €$ & $11.399,70 €$ \\
\hline Etanercept + MTX & 4 jeringas/ $100 \mathrm{mg}$ & 25 & 1 & $109,52 €$ & $109,52 €$ & $11.390,08 €$ \\
\hline Adalimumab + MTX & 2 jeringas/ $80 \mathrm{mg}$ & 40 & 1 & $475,58 €$ & $475,58 €$ & $12.365,08 €$ \\
\hline Golimumab + MTX & 1 jeringa/ $50 \mathrm{mg}$ & 50 & 1 & $1.033,23 €$ & $1.033,23 €$ & $12.398,76 €$ \\
\hline Abatacept + MTX & $1 \mathrm{vial} / 250 \mathrm{mg}$ & 750 & 3 & $309,71 €$ & $929,13 €$ & $12.078,69 €$ \\
\hline Infliximab $(3 \mathrm{mg} / \mathrm{kg})+$ MTX & $1 \mathrm{vial} / 100 \mathrm{mg}$ & 300 & 3 & $496,06 €$ & $1.488,18 €$ & $9.673,17 €$ \\
\hline Infliximab $(5 \mathrm{mg} / \mathrm{kg})+$ MTX & $1 \mathrm{vial} / 100 \mathrm{mg}$ & 400 & 4 & $496,06 €$ & $1.984,24 €$ & $12.897,56 €$ \\
\hline Rituximab + MTX & $1 \mathrm{vial} / 500 \mathrm{mg}$ & 1000 & 2 & $1.153,48 €$ & $2.306,95 €$ & $9.227,80 €$ \\
\hline Tocilizumab + MTX & $1 \mathrm{vial} / 80 \mathrm{mg}$ & 560 & 7 & $129,13 €$ & $903,91 €$ & $11.750,83 €$ \\
\hline
\end{tabular}

Fuente: elaboración propia. Datos EMA [5]. Oblikue Consulting 2013 [20]. BOT Pharma [17]. MSSSI [18]. PVL: Precio Venta Público; descuento obligatorio RD 8/2010: Descuento obligatorio aplicable a estos fármacos según el Real-Decreto Ley 8/2010; MTX: metotrexato; mg: miligramos; Uds: unidades

${ }^{a}$ El coste anual aquí reflejado es el de mantenimiento y por tanto no considera los costes de inducción

Los costes de los fármacos intravenosos, considerados en el caso base, han sido por unidad, de tal manera que se imputó todo el coste del vial abierto o de la jeringa, aunque no sea administrado al paciente en su totalidad, asumiendo así que no existe optimización del contenido de los distintos viales y jeringas precargadas. El régimen de dosis y la frecuencia de administración de los fármacos están basados en las características especificadas en las fichas técnicas de cada producto [5]. El coste farmacológico de las terapias consideradas está ligado a la dosis administrada. Por ello, las dosis para las terapias con certolizumab pegol, adalimumab, etanercept, golimumab y rituximab fueron independientes del peso de los pacientes, mientras que para las dosis de infliximab, abatacept y tocilizumab se calcularon según la masa corporal del paciente considerando un peso medio de $70 \mathrm{~kg}$ en el caso base.

\section{Costes de administración y formación}

Para estimar los costes de administración y formación, en el caso de inyecciones subcutáneas (s.c.) —certolizumab pegol, adalimumab, golimumab y etanercept-, se asume que los pacientes reciben una hora de asesoría por parte de una enfermera y que, desde ese momento, son capaces de autoadministrarse el fármaco en su domicilio sin necesidad de asistencia médica. Para todos los agentes subcutáneos suponemos que el coste de formación por paciente es único e idéntico (igual a 14,44 EUR, el coste de una consulta de enfermera especializada [20]), y que los costes de administración, son iguales a cero (Tabla 3).
Para fármacos administrados mediante infusión i.v. (abatacept, infliximab, tocilizumab y rituximab) se ha utilizado un coste medio de 254,68 EUR [20] por administración y paciente, y el tratamiento se recibe en atención hospitalaria ambulatoria en hospital de día. El número medio de administraciones anuales se calcula dividiendo el número total de administraciones del período entero de tratamiento entre el número de años en tratamiento. El número total de administraciones en los primeros seis meses y en cada período posterior de seis meses, así como el promedio de la duración del tratamiento provienen de la literatura publicada [5]. Esta estimación se aplica en el modelo al número de pacientes que reciben un tratamiento específico en un año dado. En este sentido, es importante resaltar que el modelo presenta resultados del coste anual por paciente en las fases de inducción (primer año) y mantenimiento (promedio de los siguientes), a través de los que se calcula el coste medio de administración y formación para los fármacos de infusión i.v. (Tabla 3).

\section{Costes de monitorización (visitas médicas y análisis clínicos)}

El régimen de monitorización de los fármacos proviene de la literatura [21, 22] (Tabla 4). El esquema de monitorización para los nuevos FAME biológicos de inyección s.c. (certolizumab pegol y golimumab), tanto en las visitas médicas como en los análisis clínicos, se asumió idéntico al de etanercept y adalimumab. El número de visitas médicas de monitorización para los pacientes tratados con fármacos administrados por infusión i.v. (rituximab, abatacept, tocilizumab 
Tabla 3 Resumen de los costes medios de administración y formación por paciente/año según terapia

\begin{tabular}{|c|c|c|c|c|c|c|c|c|}
\hline \multicolumn{4}{|l|}{ Subcutáneos } & \multicolumn{5}{|l|}{ Infusión i.v. } \\
\hline $\begin{array}{l}\text { Certolizumab } \\
\text { pegol + MTX }\end{array}$ & Etanercept + MTX & $\begin{array}{l}\text { Adalimumab } \\
+ \text { MTX }\end{array}$ & $\begin{array}{l}\text { Golimumab } \\
+ \text { MTX }\end{array}$ & $\begin{array}{l}\text { Abatacept } \\
+ \text { MTX }\end{array}$ & $\begin{array}{l}\text { Infliximab } \\
(3 \mathrm{mg} / \mathrm{kg}) \\
+ \text { MTX }\end{array}$ & $\begin{array}{l}\text { Infliximab } \\
(5 \mathrm{mg} / \mathrm{kg}) \\
+ \text { MTX }\end{array}$ & $\begin{array}{l}\text { Rituximab } \\
+ \text { MTX }\end{array}$ & $\begin{array}{l}\text { Tocilizumab } \\
+ \text { MTX }\end{array}$ \\
\hline $14,44 €$ & $14,44 € €$ & $14,44 €$ & $14,44 €$ & $3.393,53 €$ & $1.841,47 €$ & $1.841,47 €$ & $1.018,72 €$ & $3.056,16 €$ \\
\hline
\end{tabular}

Fuente: Elaboración propia. i.v.: intravenosa; MTX: metotrexato; mg: miligramos; kg: kilogramos

e infliximab) se asumió igual a cero. Para tratamientos i.v., suponemos que la monitorización asociada al MTX tiene lugar durante las visitas en las que se administra el tratamiento.

Los costes del personal sanitario, de las visitas médicas, los análisis y pruebas diagnósticas proceden de información oficial sobre gasto sanitario para el año 2013 [20]. De acuerdo con estas fuentes, la visita a un médico de atención primaria tiene un coste medio de 27,68 EUR, el coste de una enfermera de hospital es de 14,44 EUR, la primera visita a un reumatólogo 109,34 EUR y las sucesivas 65,61 EUR, y el coste de un farmacéutico hospitalario 128,57 EUR.

El coste anual medio de monitorización por paciente se calcula dividiendo el coste total de la monitorización en el período entero de tratamiento entre el número de años en tratamiento (Tabla 4). El coste total de la monitorización para el período entero del tratamiento se calcula a partir del número de pacientes en los primeros seis meses de tratamiento y en cada período de seis meses posterior.

Los costes directos sanitarios totales en el primer año y años posteriores equivalen a la suma de los costes de adquisición de las terapias farmacológicas, su administración y monitorización. Los costes anuales promedio de cada FAME biológico se calculan multiplicando los costes totales por el porcentaje de pacientes susceptibles de ser tratados con FAME biológicos en cada año del análisis.

Con la introducción de certolizumab pegol en el año uno, todos los pacientes que reciben certolizumab pegol están en su primer año de tratamiento, con lo que los costes de IP se calculan multiplicando los costes estimados del primer año por el número de pacientes que recibe certolizumab pegol. En los años dos, tres, cuatro y cinco, se utilizan los costes anuales medios para calcular los costes del impacto de la misma forma que para los demás FAME biológicos.

Todos los costes referidos anteriormente son actualizados a precios de 2013 a partir de las estadísticas oficiales del Índice de Precios al Consumo (IPC) [23].

Estimación de la cuota de mercado. Caso base

La cuota de mercado estimada para certolizumab pegol se basa en pronósticos de mercado de la compañía comercializadora UCB. Las cuotas de mercado de las demás terapias se basan en datos de investigación de mercado de la compañía, validadas por el panel de expertos previamente citado, y se presentan en términos de pacientes tratados. En el escenario base, suponemos que certolizumab pegol reemplaza otros FAME biológicos s.c. o i.v. de forma proporcional a la cuota de mercado. Se estima que en el año 2013 certolizumab pegol tiene una cuota total de mercado de $8,90 \%$, y que ésta aumentará progresivamente hasta alcanzar 17,7\% en 2017 (Tabla 5).

Análisis de sensibilidad

Todo modelo económico lleva asociado cierto nivel de incertidumbre relativo a posibles variaciones en los supuestos asumidos en el caso base. Para analizar la solidez de los resultados, realizamos un análisis de sensibilidad univariante con aquellos parámetros del modelo que se considera que tienen una mayor incertidumbre asociada, variando los valores adoptados en el caso base. Los parámetros analizados fueron los seis siguientes: la cuota de mercado de certolizumab pegol $( \pm 50 \%)$, los costes de administración y formación de las terapias i.v. $( \pm 50 \%)$, los costes de monitorización de todas las terapias biológicas $( \pm 50 \%)$, el peso medio de los pacientes $( \pm 10 \mathrm{~kg})$ y la tasa de descuento anual sobre los costes (1\% y 5\%). Además, analizamos la repercusión que tendría sobre el IP una variación en el descuento obligatorio aplicable a los precios de todos los fármacos, a partir del escenario base $( \pm 50 \%)$.

\section{Resultados}

El IP anual se estimó como la diferencia entre el coste farmacológico, de administración y de monitorización del manejo de los pacientes tratados con certolizumab pegol y el coste que habría supuesto su tratamiento con otras terapias biológicas.

En el escenario base, la utilización del certolizumab pegol en el mercado español de FAME biológicos generaría un ahorro presupuestario estimado en 10,26 millones EUR distribuidos a lo largo del período 2013-2017. Esta cifra equivale al $0,59 \%$ del coste en dicho período atribuible a las tera- 
Tabla 4 Coste medio de monitorización por paciente/año y regímenes de monitorización según terapia

\begin{tabular}{|c|c|c|c|c|c|c|c|c|c|}
\hline & & \multicolumn{4}{|l|}{ Subcutáneos } & \multicolumn{4}{|l|}{ Infusión i.v. } \\
\hline & & $\begin{array}{l}\text { Certolizumab } \\
\text { pegol + MTX }\end{array}$ & $\begin{array}{l}\text { Etanercept } \\
+ \text { MTX }\end{array}$ & $\begin{array}{l}\text { Adalimumab } \\
+ \text { MTX }\end{array}$ & $\begin{array}{l}\text { Golimumab } \\
+ \text { MTX }\end{array}$ & $\begin{array}{l}\text { Abatacept } \\
+ \text { MTX }\end{array}$ & $\begin{array}{l}\text { Infliximab } \\
+ \text { MTX }\end{array}$ & $\begin{array}{l}\text { Rituximab } \\
+ \text { MTX }\end{array}$ & $\begin{array}{l}\text { Tocilizumab } \\
+ \text { MTX }\end{array}$ \\
\hline \multirow{6}{*}{$\begin{array}{l}\text { Coste medio } \\
\mathrm{N}^{\mathrm{o}} \text { de } \\
\text { pruebas } \\
\text { antes del } \\
\text { tratamiento }\end{array}$} & o de monitorización & $683 €$ & $683 €$ & $683 €$ & $683 €$ & $198 €$ & $205 €$ & $198 €$ & $198 €$ \\
\hline & Hemograma & & & 1 & & & & 1 & \\
\hline & $\begin{array}{l}\text { Velocidad de } \\
\text { sedimentación } \\
\text { (VS) }\end{array}$ & & & 1 & & & & 1 & \\
\hline & $\begin{array}{l}\text { Perfil bioquímico } \\
\text { (PBQ) }\end{array}$ & & & 1 & & & & 1 & \\
\hline & $\begin{array}{l}\text { Radiografía de } \\
\text { tórax (RT) }\end{array}$ & & & 1 & & & & 1 & \\
\hline & Análisis de orina & & & 0 & & & & 0 & \\
\hline \multirow{7}{*}{$\begin{array}{l}\mathrm{N}^{\mathrm{o}} \text { de } \\
\text { pruebas y } \\
\text { visitas } \\
\text { médicas. } \\
\text { Primeros } \\
\text { seis meses }\end{array}$} & Hemograma & & & 10,7 & & & & 10,7 & \\
\hline & $\begin{array}{l}\text { Velocidad de } \\
\text { sedimentación } \\
\text { (VS) }\end{array}$ & & & 5,1 & & & & 4,5 & \\
\hline & $\begin{array}{l}\text { Perfil bioquímico } \\
\text { (PBQ) }\end{array}$ & & & 10,7 & & & & 10,7 & \\
\hline & $\begin{array}{l}\text { Radiografía de } \\
\text { tórax (RT) }\end{array}$ & & & 0 & & & & 0 & \\
\hline & Análisis de orina & & & 0 & & & & 0 & \\
\hline & Visitas AP & & & 12 & & & & 0 & \\
\hline & $\begin{array}{l}\text { Visitas } \\
\text { Hospitalaria }\end{array}$ & & & 1 & & & & 0 & \\
\hline \multirow{7}{*}{$\begin{array}{l}\mathrm{N}^{\mathrm{o}} \text { de } \\
\text { pruebas y } \\
\text { visitas } \\
\text { médicas. } \\
\text { Seis meses } \\
\text { posteriores }\end{array}$} & Hemograma & & & 6 & & & & 6 & \\
\hline & $\begin{array}{l}\text { Velocidad de } \\
\text { sedimentación } \\
\text { (VS) }\end{array}$ & & & 2 & & & & 3,25 & \\
\hline & $\begin{array}{l}\text { Perfil bioquímico } \\
\text { (PBQ) }\end{array}$ & & & 6 & & & & 6 & \\
\hline & $\begin{array}{l}\text { Radiografía de } \\
\text { tórax (RT) }\end{array}$ & & & 0 & & & & 0 & \\
\hline & Análisis de orina & & & 0 & & & & 0 & \\
\hline & Visitas AP & & & 5 & & & & 0 & \\
\hline & $\begin{array}{l}\text { Visitas } \\
\text { Hospitalaria }\end{array}$ & & & 1 & & & & 0 & \\
\hline
\end{tabular}

Fuente: Brennan, A., Bansback, N., Nixon, R. [21]. MTX: metotrexato. Chen, Y.F. et al. [22], Oblikue Consulting 2013 [20]. s.c.: subcutánea; i.v.: intravenosa; AP: Atención Primaria

pias biológicas en pacientes con AR de moderada a grave en España (1.747 millones EUR). Los resultados indican que la utilización de certolizumab pegol genera cuantiosos ahorros para el SNS cuando sustituye a terapias como abatacept, infliximab (3 y $5 \mathrm{mg} / \mathrm{kg}$ ), tocilizumab, etanercept, golimumab y adalimumab en combinación con MTX.

La Tabla 6 muestra que los ahorros más cuantiosos provienen de la reducción en costes de administración. Se percibe también un aumento en el coste farmacológico y el coste de monitorización que disminuye los ahorros anteriores. Cabe señalar que los ahorros generados en términos de cos- tes de administración van ganando relevancia progresivamente a medida que pasa el tiempo. En efecto, con la utilización de certolizumab pegol en 2013 los ahorros en términos de administración de los fármacos serían de 1,83 millones EUR, mientras que en 2017 se espera que alcancen los 5,55 millones EUR (más del 100\% del ahorro total esperado para ese año de 4,3 millones EUR).

Resultados del análisis de sensibilidad

El análisis de sensibilidad univariante realizado (Fig. 2; Tabla 7) muestra que si la cuota de mercado de certolizumab 
Tabla 5 Cuota de mercado estimada para certolizumab pegol y otros FAME biológicos

Fuente: Elaboración propia a partir de los datos de investigación de mercado de UCB Pharma y del panel de expertos. FAME: fármaco antirreumático modificador de la enfermedad; MTX: metotrexato; $\mathrm{mg} / \mathrm{kg}$ : miligramos/kilogramo de peso

\begin{tabular}{lrrrrr}
\hline Intervención & \multicolumn{1}{c}{2013} & \multicolumn{1}{c}{2014} & \multicolumn{1}{c}{2015} & \multicolumn{1}{c}{2016} & 2017 \\
\hline Certolizumab pegol + MTX & $8,90 \%$ & $10,70 \%$ & $13,00 \%$ & $15,70 \%$ & $17,70 \%$ \\
Etanercept + MTX & $22,80 \%$ & $20,83 \%$ & $18,82 \%$ & $16,80 \%$ & $15,00 \%$ \\
Abatacept + MTX & $5,50 \%$ & $6,11 \%$ & $6,64 \%$ & $7,11 \%$ & $7,60 \%$ \\
Infliximab (3 mg/kg) + MTX & $5,50 \%$ & $4,78 \%$ & $4,05 \%$ & $3,35 \%$ & $2,70 \%$ \\
Infliximab (5 mg/kg) + MTX & $5,50 \%$ & $4,78 \%$ & $4,05 \%$ & $3,35 \%$ & $2,70 \%$ \\
Rituximab + MTX & $9,40 \%$ & $8,08 \%$ & $6,76 \%$ & $5,48 \%$ & $4,30 \%$ \\
Tocilizumab + MTX & $12,50 \%$ & $12,96 \%$ & $13,32 \%$ & $13,57 \%$ & $13,90 \%$ \\
Adalimumab (cada 2 semanas) + MTX & $24,70 \%$ & $25,10 \%$ & $25,33 \%$ & $25,38 \%$ & $25,60 \%$ \\
Golimumab + MTX & $5,20 \%$ & $6,67 \%$ & $8,03 \%$ & $9,27 \%$ & $10,50 \%$ \\
\hline
\end{tabular}

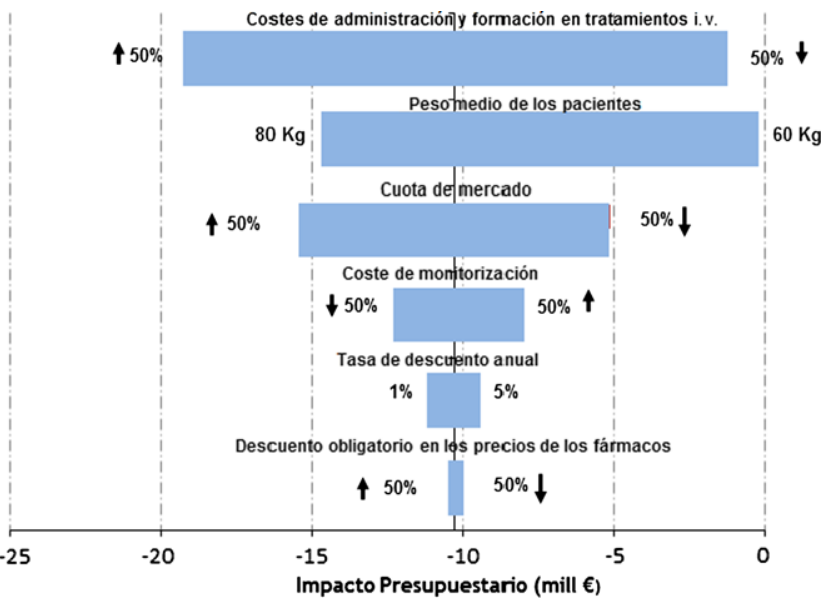

Tabla 6 Presupuesto destinado a FAME biológicos, SIN y CON la introducción de certolizumab pegol e impacto presupuestario consecuente (millones EUR), por tipo de coste (2013-2017)

\begin{tabular}{lccr}
\hline COSTE DEL & $\begin{array}{l}\text { Sin } \\
\text { TRATAMIENTO } \\
\text { pegol (A) }\end{array}$ & $\begin{array}{l}\text { Con } \\
\text { certolizumab } \\
\text { pegol }(\mathrm{B})\end{array}$ & $\begin{array}{r}\text { Diferencia } \\
(\mathrm{B}-\mathrm{A}) \mathrm{IP}\end{array}$ \\
\hline Fármaco & $1.560,82$ & $1.564,45$ & 3,63 \\
Administración & 131,02 & 113,23 & $-17,79$ \\
Monitorización & 65,35 & 69,25 & 3,90 \\
TOTALES & $1.757,20$ & $1.746,94$ & $-10,26$ \\
\hline
\end{tabular}

Fuente: Elaboración propia a partir de los resultados del modelo. IP: impacto presupuestario; FAME: fármaco antirreumático modificador de la enfermedad

pegol, en cada uno de los años considerados, aumentase en un 50\%, la introducción de este fármaco generaría un ahorro agregado de 15,38 millones EUR en el período 2013-2017 ( $0,83 \%$ del presupuesto destinado a terapias biológicas). En el caso contrario, ante una disminución del $50 \%$ en las cuotas de mercado estimadas para certolizumab pegol, el ahorro sería de 5,13 millones EUR para el mismo período. Ante variaciones en los costes de administración y formación de las infusiones i.v. (los de las terapias subcutáneas se asumen iguales a cero), si estos aumentasen un 50\%, el ahorro sería de 19,18 millones EUR, mientras que si disminuyesen un $50 \%$ el ahorro sería de 1,33 millones EUR. En cuanto a los costes de monitorización de todos los fármacos, si estos aumentasen un 50\%, el ahorro alcanzaría 8,30 millones EUR. Si disminuyesen un 50\%, el ahorro sería de 12,21 millones EUR, ya que en este caso el coste afecta a todos los medicamentos y también a certolizumab pegol.

Dado que la dosis administrada de infliximab, abatacept y tocilizumab está en función del peso del paciente, evaluamos el IP asociado a variaciones en el peso medio de los pacientes, manteniendo los demás supuestos del caso base. La Fig. 3 muestra una asociación negativa entre peso e IP: a mayor peso medio de los pacientes, mayor ahorro esperado. Existe un IP positivo para pesos medios inferiores a $58 \mathrm{~kg}$.
Figura 2 Resultados del análisis de sensibilidad: gráfico tipo tornado. Fuente: Elaboración propia. Impacto presupuestario en millones de euros. (Un signo negativo indica ahorros para el SNS.) Kg: kilogramos

A partir de ese punto de inflexión, se registran ahorros crecientes a tasas estables hasta $80 \mathrm{~kg}$.

En cuanto a las posibles variaciones del descuento obligatorio sobre PVL de los fármacos, las variaciones del IP observadas son mínimas, siendo los ahorros de 10,40 millones EUR y 10,11 millones EUR, ante cambios en el descuento obligatorio sobre PVL de $\pm 50 \%$, respectivamente.

Una disminución de la tasa de descuento, pasando la misma del $3 \%$ al 1\%, supondría un mayor ahorro para el SNS de 0,67 millones EUR, siendo este efecto el contrario (menor ahorro por un total de 0,62 millones EUR) al aumentar la tasa de descuento del 3\% al 5\%. En ambos casos el IP se

Por tanto, el análisis de sensibilidad muestra la robustez de los resultados del análisis realizado para el caso base ante variaciones tanto en costes relevantes, como en otros parámetros importantes como cuota de mercado esperada, peso medio de los pacientes y tasa de descuento de los costes. mantendría negativo, lo que indicaría un ahorro para el SNS. 
Tabla 7 Resultados del análisis de sensibilidad univariante

Fuente: Elaboración propia a partir de los resultados del modelo. i.v.: intravenosa; RD 8/2010: Descuento obligatorio aplicable a estos fármacos según el Real-Decreto Ley 8/2010

${ }^{a}$ Aquellas terapias que NO se ajustan según masa corporal

\begin{tabular}{lll}
\hline Parámetro & \multicolumn{2}{l}{$\begin{array}{l}\text { Porcentaje de variación del parámetro } \\
\text { considerado en el caso base }\end{array}$} \\
\cline { 2 - 3 } & $+50 \%$ & $-50 \%$ \\
\hline $\begin{array}{l}\text { Costes de administración y formación en } \\
\text { terapias i.v. }\end{array}$ & $-19,18$ millones $€$ & $-1,33$ millones $€$ \\
Costes de monitorización & $-8,30$ millones $€$ & $-12,21$ millones $€$ \\
Cuota de mercado de certolizumab pegol & $-15,38$ millones $€$ & $-5,13$ millones $€$ \\
$\begin{array}{l}\text { Descuentos obligatorio en el precio de } \\
\text { todas las terapias (RD 8/2010) }\end{array}$ & $-10,40$ millones $€$ & $-10,11$ millones $€$ \\
Parámetro & Variación & \\
Caso base 70 kg & & $\mathbf{+ 1 0}$ kg (80 kg) \\
Peso medio del paciente & $-14,67$ millones $€$ & $-\mathbf{1 0 ~ k g ~ ( 6 0 ~ k g ) ~}$ \\
Parámetro & Variación & $-0,23$ millones $€$ \\
Caso base 3\% & $\mathbf{1 \%}$ & $\mathbf{5 \%}$ \\
Tasa de descuento anual & $-10,93$ millones $€$ & $-9,64$ millones $€$ \\
\hline
\end{tabular}

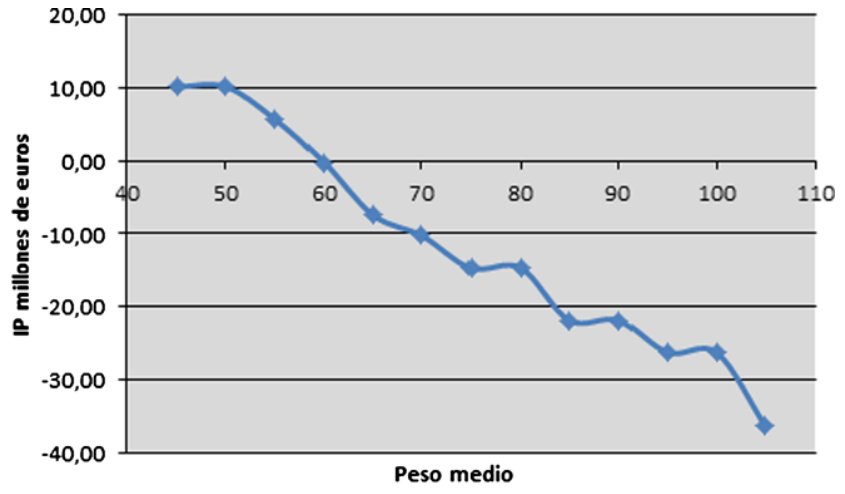

Figura 3 Evaluación del Impacto presupuestario en función del peso de los pacientes. Fuente: Elaboración propia. Kg: kilogramos

Impacto presupuestario por Comunidad Autónoma

El análisis del IP a nivel de CCAA muestra cómo se distribuye el ahorro esperado entre las Consejerías de Sanidad tras la introducción de certolizumab pegol. En el caso base, este oscila entre 0,01 millones EUR (Ceuta y Melilla) y 1,71 millones EUR (Andalucía) a lo largo del período 2013-2017. Los mayores ahorros proyectados son para las Consejerías de Sanidad de Andalucía, Cataluña (1,60 millones EUR) y Madrid (1,38 millones EUR).

\section{Discusión}

Este análisis de IP muestra que la introducción paulatina de certolizumab pegol en España generaría un ahorro en el presupuesto del SNS, cuya magnitud depende fundamentalmente de los supuestos empleados sobre la cuota de mercado para certolizumab pegol, los costes de administración y formación de las infusiones i.v., y los costes de monitorización de todas las terapias biológicas, a lo largo de un horizonte temporal de cinco años. En el caso base se estima un ahorro presupuestario agregado de 10,26 millones EUR, lo que representa un $0,59 \%$ del coste atribuible en dicho período a las terapias biológicas en pacientes con AR de moderada a grave en España (1.747 millones EUR). Los ahorros en términos de costes de administración de los fármacos van ganando relevancia progresivamente en el período considerado. Esto se debe a que los costes de administración de certolizumab pegol son iguales a cero, mientras que los de las terapias i.v. que reemplazaría son siempre positivos. Durante el primer año del análisis (debido a las dosis de inducción) la terapia con certolizumab pegol puede generar un IP positivo puntual, si bien estos costes adicionales van siendo compensados por los ahorros en términos de costes de administración durante los siguientes años (mantenimiento). En efecto, una de las razones que justificarían el ahorro potencial asociado a la utilización de certolizumab pegol es su administración subcutánea, que evita los costes asociados a las infusiones i.v. (254,68 EUR por administración). Existe la posibilidad de variaciones importantes en las estimaciones del coste de la administración i.v. debido a que ciertos FAME biológicos requieren un menor tiempo de infusión i.v. Sin embargo, el análisis de sensibilidad realizado muestra que incluso una disminución del $50 \%$ en estos costes generaría ahorros por 1,33 millones EUR en el presupuesto en el período analizado.

Por otro lado, el peso medio de los pacientes asumido en el caso base $(70 \mathrm{~kg})$ podría estar subestimando el verdadero peso de los pacientes. El peso medio en ensayos clínicos con certolizumab pegol fue de $81,4 \mathrm{~kg}$. Si esto fuese así, el IP obtenido en el caso base corresponde a un escenario conservador y los ahorros podrían ser mayores, como mostró la evaluación del IP en función de la masa corporal de los pacientes. 
Cabe señalar que un análisis IP en el que se asumiera que todos los pacientes tratados con infliximab reciben la dosis máxima $(10 \mathrm{mg} / \mathrm{kg})$, los tratados con adalimumab reciben dosis semanales (en vez de cada dos semanas) y los tratados con rituximab reciben un ciclo cada 6 meses, generaría resultados aún más favorables para la introducción de certolizumab pegol.

Es posible que existan ahorros adicionales a los aquí estimados debidos a la introducción de certolizumab pegol en el SNS español. En los estudios clínicos de certolizumab pegol [6-9], se observaron reducciones significativas en los signos radiológicos indicativos de disminución de progresión de la enfermedad con certolizumab pegol + MTX en las semanas 24 (RAPID 1 y RAPID 2) y 52 (RAPID 1) frente al uso de MTX en monoterapia. Esto puede llevar a una reducción en el número de consultas y estancias hospitalarias relacionadas con el reemplazo de articulaciones, y por ende a un ahorro en el uso de recursos hospitalarios, aunque esto último no está demostrado todavía.

\section{Limitaciones del modelo}

Como todos los modelos, este de IP tiene ciertas limitaciones. La mayor parte de las limitaciones imputables al uso de asunciones han sido abordadas mediante el análisis de sensibilidad realizado para comprobar que el modelo es robusto y determinar el impacto en función de cambios en las variables más sensibles al resultado final. Sin embargo, existen otro tipo de limitaciones en el modelo sobre las que no hemos podido rebajar la incertidumbre y que debemos tener en consideración. La primera es que este no deja de ser un modelo de proyección futura de uso de un medicamento, basado en asunciones múltiples y en la actitud esperada de los clínicos ante la introducción de certolizumab pegol en el mercado de FAME biológicos. Si dicha actitud difiere de la esperada, las cuotas de mercado para certolizumab pegol podrían ser diferentes a las analizadas en este estudio. No obstante, el análisis de sensibilidad ha demostrado que aun variando significativamente las cuotas de mercado esperadas $( \pm 50 \%)$, el ahorro para el SNS sigue siendo importante tras la introducción de certolizumab pegol.

En segundo lugar, debido a la falta de datos publicados sobre la frecuencia de ingresos hospitalarios y artroplastias, este IP no incorpora supuestos de ahorros en costes referentes a estas partidas presupuestarias. Sin embargo, estos ahorros se repartirían entre todos los FAME biológicos de forma más o menos proporcional a su cuota de mercado.

En tercer lugar, la asunción de que la proporción de pacientes en primer año de tratamiento es constante e igual al $10 \%$ y de que los pacientes permanecen los cinco años en tratamiento, sin considerar una tasa de abandonos por acontecimientos adversos, muertes, etc., implica un cierto alejamiento de la realidad clínica. Sin embargo, a efectos de IP, este supuesto es neutral ya que afecta por igual a todos los fármacos considerados.

En cuarto lugar, la asunción de que no existe incremento de dosis de ningún fármaco a lo largo de los cinco años del análisis es poco realista en la práctica clínica, especialmente con determinados fármacos como infliximab por ejemplo, lo que redundaría en mayores ahorros en este IP.

En quinto lugar, hemos asumido que no existe optimización de viales en tratamientos i.v. Si hubiese dicha optimización, el ahorro podría ser menor ante la introducción de certolizumab pegol.

Las dos últimas limitaciones afectarían a las cifras finales del modelo, una con signo positivo, suponiendo mayores ahorros para el SNS (la cuarta) y la otra negativamente (la quinta). Sin embargo, creemos que dichos efectos se podrían compensar entre sí y que, por lo tanto, las cifras que concluimos en nuestro análisis muestran el rango real de ahorro para el SNS español que supondría la introducción de certolizumab pegol.

\section{Conclusiones}

Aun con las limitaciones anteriormente expuestas, el análisis concluye que la utilización de certolizumab pegol en el mercado español de FAME biológicos para el tratamiento de pacientes con AR de moderada a grave que no muestran una respuesta adecuada a los FAME convencionales podría generar ahorros netos de 10,26 millones EUR entre 2013 y 2017 (0,59\% del coste total atribuible en dicho período). Estos ahorros provendrían fundamentalmente de la reducción en los costes de administración y la ausencia de necesidad de intensificación de dosis o ajustes por peso del paciente. El IP esperado puede variar dependiendo fundamentalmente de los supuestos empleados sobre los costes de administración y formación de terapias administradas por infusión i.v., los costes de monitorización de todos los fármacos, la cuota de mercado para certolizumab pegol y el peso medio de los pacientes. Sin embargo, el análisis es suficientemente robusto y muestra ahorros para el SNS español ante variaciones importantes en las variables anteriores. Lo que demuestra que disponer de alternativas terapéuticas, además de incrementar las posibilidades para el tratamiento de los pacientes y para los profesionales del SNS, contribuye a la sostenibilidad del propio SNS.

En base a estos ahorros, los servicios de salud autonómicos y nacionales deberían promover la elección de estrategias terapéuticas cómodas, eficientes y coste-efectivas fundamentalmente en patologías crónicas como la artritis reumatoide, que aseguren el cumplimiento del tratamiento a largo plazo y favorezcan el control de las patologías.

Agradecimientos Queremos agradecer los comentarios de Ángel Sanz, gerente de farmacoeconomía de Weber Economía y Salud, y la ayuda estadística de Almudena González del IMW. 
Financiación y conflicto de intereses La realización del presente estudio ha sido posible gracias a la financiación no condicionada de UCB, España. Todos los autores declaran que el análisis ha sido realizado de forma independiente y los resultados obtenidos son neutrales.

Pablo Talavera y Belén Ferro son empleados de UCB, España. Álvaro Hidalgo, Renata Villoro y Alexandra Ivanova son investigadores de la UCLM y del Instituto Max Weber, respectivamente. Esta última ha sido la entidad empleada por UCB para la adaptación del modelo, libre y no condicionada. El resto de los autores declaran no presentar conflicto de intereses.

Open Access This article is distributed under the terms of the Creative Commons Attribution License which permits any use, distribution, and reproduction in any medium, provided the original author(s) and the source are credited.

\section{Bibliografía}

1. Mattey DL, Thomson W, Ollier WE, Batley M, Davies PG, Gough AK, et al. Association of DRB1 shared epitope genotypes with early mortality in rheumatoid arthritis: results of eighteen years of followup from the early rheumatoid arthritis study. Arthritis Rheumatol. 2007;56(5):1408-16.

2. Tornero J, Sanmartí R, Rodríguez-Valverde V, Martín E, Marenco de la Fuente J, González A, et al. Actualización del documento de Consenso de la Sociedad Española de Reumatología sobre el uso de la terapias biológicas en la artritis reumatoide. Reumatol Clin. 2010;6(1):23-36.

3. Blanco FJ, Ballina J, Carbonell J, Martín-Mola E, Tornero J, Ramírez E, et al. Descriptive study of the use of DMARD in patients with rheumatoid arthritis or persistent arthritis who start drug treatment in Spain (FIRST). Reumatol Clin. 2013;7(2):8893.

4. Genovese MC, Bathon JM, Martin RW, Fleischmann RM, Tesser JR, Schiff MH, et al. Etanercept versus methotrexate in patients with early rheumatoid arthritis: two-year radiographic and clinical outcomes. Arthritis Rheumatol. 2002;46(6):1443-50.

5. EMA. European Medicines Agency (EMA). EPARs for authorised medicinal products for human use. Disponible en: http://www.ema.europa.eu/ema/index.jsp?curl=pages/medicines/ landing/epar_search.jsp\&mid=WC0b01ac058001d124.

6. Keystone E, Heijde D, Mason D Jr, Landewe R, Vollenhoven RV, Combe B, et al. Certolizumab pegol plus methotrexate is significantly more effective than placebo plus methotrexate in active rheumatoid arthritis: findings of a fifty-two-week, phase III, multicenter, randomized, double-blind, placebo-controlled, parallelgroup study. Arthritis Rheumatol. 2008;58(11):3319-29.

7. Keystone EC, Combe B, Smolen J, Strand V, Goel N, van Vollenhoven R, et al. Sustained efficacy of certolizumab pegol added to methotrexate in the treatment of rheumatoid arthritis: 2-year results from the RAPID 1 trial. Rheumatol (Oxford). 2012;51(9):1628-38.

8. Smolen J, Landewe RB, Mease P, Brzezicki J, Mason D, Luijtens $\mathrm{K}$, et al. Efficacy and safety of certolizumab pegol plus methotrexate in active rheumatoid arthritis: the RAPID 2 study. A randomised controlled trial. Ann Rheum Dis. 2009;68(6):797804.
9. Fleischmann R, Vencovsky J, Vollenhoven R, et al. Efficacy and safety of certolizumab pegol monotherapy every 4 weeks in patients with rheumatoid arthritis failing previous disease-modifying antirheumatic therapy: the rheumatoid arthritis failing previous monotherapy every 4 weeks in patients with efficacy. Ann Rheum Dis. 2009;68:805-11.

10. Mauskopf JA, Sullivan SD, Annemans L, Caro J, Mullins CD, Nuijten M, et al. Principles of good practice for budget impact analysis: report of the ISPOR Task Force on good research practices-budget impact analysis. Value Health. 2007;10(5):336-47.

11. Brosa M, Gisbert R, Rodríguez JM, Soto J. Principios, métodos y aplicaciones del análisis de impacto presupuestario en el sector sanitario. PharmacoEcon Spain Res Art. 2005;2:65-79.

12. INE. Instituto Nacional de Estadística (INE). Estimaciones de la Población Actual. Disponible en: http://www.ine.es/jaxi/menu. do?type $=$ pcaxis $\&$ path $=\% 2 \mathrm{Ft} 20 \sim \% 2 \mathrm{Fp} 259 \&$ file $=$ inebase $\& \mathrm{~L}=0$.

13. ENS. Instituto Nacional de Estadística (INE). Encuesta Nacional de Salud (ENS). Año 2006: Problemas o enfermedades crónicas o de larga evolución diagnosticadas por un médico por sexo y grupo de edad. Disponible en: http://www.ine.es/ss/Satellite $? \mathrm{c}=$ TFichaIOE_C\&cid=0\&p=1254735038414\&pagename $=$ IOEhist\%2FIOEhistLayout \&L=0\&param 1=54009.

14. SER. Sociedad Española de Reumatología: Informe de prevalencia de enfermedades reumáticas. Disponible en: http://www.ser.es/ investigacion/Informes_Estadisticos.ph.

15. INE. Instituto Nacional de Estadística (INE). Proyecciones de población a corto plazo 2009-2019: Resultados por Comunidad Autónoma. Disponible en: http://www.ine.es/jaxi/menu.do?type= pcaxis \&path $=\% 2 \mathrm{Ft} 20 \sim \% 2 \mathrm{Fp} 259 \&$ file $=$ inebase $\& \mathrm{~L}=0$.

16. Datamonitor. Stakeholder insight: rheumatoid arthritis—biologics battle up the treatment algorithm. Sep 2006.

17. BOTfarma. BOT Base de datos del medicamento. Consejo General de Colegios Oficiales de Farmacéuticos. Disponible en: https://botplusweb.portalfarma.com/.

18. MSSSI. Información sobre los productos incluidos en la prestación farmacéutica del SNS (dispensables a través de oficinas de farmacia): Nomenclátor de Facturación de Diciembre/2013. Disponible en: http://www.msc.es/profesionales/nomenclator.do.

19. BOE 210/05/24. Real Decreto-ley 8/2010, de 20 de mayo, por el que se adoptan medidas extraordinarias para la reducción del déficit público. Disponible en: http://www.boe.es/boe/ dias/2010/05/24/pdfs/BOE-A-2010-8228.pdf.

20. Oblikue. eSalud-Información económica del sector sanitario. Oblikue Consulting S.L. Disponible: http://oblikue.com/ bddcostes/. Consultado: marzo 2013.

21. Brennan A, Bansback N, Nixon R, Madan J, Harrison M, Watson $\mathrm{K}$, et al. Modelling the cost effectiveness of TNF-alpha antagonists in the management of rheumatoid arthritis: results from the British Society for Rheumatology Biologics Registry. Rheumatol (Oxford). 2007;46(8):1345-54.

22. Chen YF, Jobanputra P, Barton P, Jowett S, Bryan S, Clark W, et al. A systematic review of the effectiveness of adalimumab, etanercept and infliximab for the treatment of rheumatoid arthritis in adults and an economic evaluation of their cost-effectiveness. Health Technol Assess. 2006;10(42):iii-iv, xi-xiii, 1-229.

23. INE. Instituto Nacional de Estadística (INE). Nivel, calidad y condiciones de vida: índice de precios al consumo. Disponible en: http://www.ine.es/jaxi/menu.do?type=pcaxis\&path=/t25/p138\& file $=$ inebase $\& \mathrm{~L}=0$. 